\title{
Using Skin Color and HAD-AdaBoost Algorithm for Face Detection in Color Images
}

\author{
Li-Jie Xue \\ School of Electronic and Information Engineering, \\ Jiangsu University \\ Zhenjiang, PR of China, 15052919053 \\ Xuelijie_2010@163.com
}

\author{
Zheng-Ming Li \\ School of Electronic and Information Engineering, \\ Jiangsu University \\ Zhenjiang, PR of China, 13705286653 \\ lzming@ujs.edu.cn
}

\begin{abstract}
Owing to the interference of the complex background in color image, high false positive rate is a problem in face detection based on AdaBoost algorithm. In addition, the training process of AdaBoost is very time consuming. To address these problems, this paper proposes a two-stage face detection method using skin color segmentation and heuristics-structured adaptive to detection AdaBoost (HAD-AdaBoost) algorithm. Firstly, skin color segmentation is applied to remove most of the background. Then, the HAD-AdaBoost cascade classifier is performed on the candidates to make a final face location. By introducing the algorithm of adaptive to detection AdaBoost algorithm (AD AdaBoost) and heuristicsstructured cascade to our system, the resulting classifier consisting of fewer weak classifiers achieves lower error rates. The experimental results demonstrate that our system retrieves $90.24 \%$ of the detection rate with $1.69 \%$ false alarms on the color image set.
\end{abstract}

Keywords-face detection; skin color segmentation; $A D$ AdaBoost; heuristics-structured cascade

\section{INTRODUCTION}

Face detection is an opening problem in research of pattern recognition and computer vision. It is widely used in many fields such as artificial intelligence, video surveillance, identity authentication, computer vision, etc [1]. In 2001, Viola and Jones proposed the first real-time face detection method based on AdaBoost with the "Integral Map" and the cascade structure, which made face detection technology practical [2]. Due to their tremendous success, numerous further works proposed. KLBoost proposed by Liu solved the problems of how to choose the best weak classifier and how to combine them [3]. $\mathrm{Li}$ proposed FloatBoost for a better detection accuracy by introducing a backward feature elimination step into the AdaBoost training procedure in 2004 [4]. Noticing the problem of time consuming, Xiao proposed boosting chain framework to shorten the total number of weak classifiers by using forward feature selection [5]. Aimed at the shortage of entirely inheriting prior classifiers in the boosting chain, Guo proposed an enhanced cascade structured Real AdaBoost algorithm by inheriting historical knowledge partly in 2007[6]. However, these Adaboost algorithms use only the gray feature of face to detect faces in color images. In image with complex

This work is partially supported by Project supported by the Priority Academic Program Development of Jiangsu Higher Education Institutions (Jiangsu provincial government general office documents [2011] No.6). background, those Adaboost algorithms may result in high false alarm rate [7]. In addition, time consuming remains a major challenge for Adaboost training.

In this paper, we propose a two-stage face detection method using both skin color features and gray features of one image. Moreover, a novel classification framework called heuristicsstructured adaptive to detection AdaBoost (HAD-AdaBoost) is proposed to reduce training time and the number of weak classifiers. AD AdaBoost which is more suitable for the cascade structure in target detection with fast weak classifier training method is applied, and heuristics-structured cascade is introduced to partly integrate historical knowledge into successive boosting learning. The first stage of the proposed face detection system is skin color segmentation, which removes most of the background area and outputs candidate skin region. Then the following HAD-AdaBoost cascade stage is performed on the candidates to filter out non-faces.

\section{METHOD}

The flow chart of the proposed method is shown in Fig. 1.

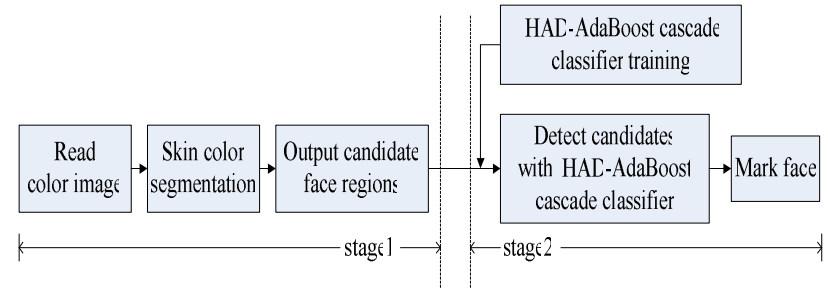

Figure 1. Flow chart of the proposed face detection system.

\section{A. Stage1: Skin color segmentation}

Skin color is not sensitive to changes in posture and rotation. Therefore, human face can be distinguished from the complex background by using skin color easily. Researches have shown that the difference between skin colors mainly concentrates in brightness and skin color distribution has the character of clustering in color space without bright. In the YCbCr space, luminance and chroma could be handled respectively and the skin color spots could form better cluster. In addition, the YCbCr space can be obtained from RGB space directly through linear transformation [8]. Moreover, "reference white" is applied to reduce the effect of lighting on segmentation performance [9]. The analysis of the skin color histogram indicates that the skin color information is distributed in a 
limited area: In the YCbCr color space, $C b \in[100,180]$ and $C r \in[135,170]$. However, some skin regions such as hands, arms, etc may be selected. We thus need to screen these regions by face shape filters:

(1) Operate morphological filter to the segmented skin regions. (2) The width and height of face region has a proportional relationship, i.e., $0.6<$ length/with $<2.0$.

\section{B. Stage2: Face detection based on HAD-AdaBoost}

In this section, we improve the Adaboost to HADAdaBoost. Firstly, we introduce AD Adaboost algorithm to balance the detection rate and the false positive rate (FPR). Then, we extend boosting chain with inheriting prior classifiers partly to be a novel framework called heuristics-structured cascade. The framework with independence characteristic and inheritance specialty can reduce the total number of the weak classier. Different from enhanced cascade algorithm in [6], the inheritance strategy of the proposed method is simpler.

In the procedure of AdaBoost, the positive samples' error rate and negative samples' error rate is equal in the training process. However, this assumption is no longer hold in detector with cascade structure. To address the problem, AD AdaBoost is proposed [10]. The weight parameters of weak classifiers in AD AdaBoost are determined not only by the FPR, but also by their abilities to recognize the positive samples. The weight parameter of the weak classifier is updated by:

$$
\alpha_{t}=\frac{1}{2} \ln \frac{1-\varepsilon_{t}}{\varepsilon_{t}}+k e^{p_{t}}
$$

Where, $k$ is a constant, $P_{t}=\sum_{y_{i}=1, h_{t}\left(x_{i}\right)=1} w_{i}$ represents the sum weights of the positive samples that be correctly identified in the $t$ th iteration, which can represent the recognition capacity of one weak classifier for the positive samples. The new weighting parameters guarantee that those weak learners which have better recognition ability for positive samples will own a larger weight in the case of the same $\varepsilon_{t}$. Then, the weight parameters of weak classifiers relate to both the overall error rate and the recognition ability for human face.

In boosting cascade, the training process of each layer is carried out independently. Although the former classifiers have discarded some negative samples, the historical information also does well to samples that were wrong classified. In this paper, heuristics-structured cascade that has an easier heuristic strategy is proposed. The heuristics-structured cascade with independence characteristic and inheritance specialty needs an evaluation parameter of the previous classifier. The schematic depiction of the heuristics-structured cascade is shown in Fig. 2.

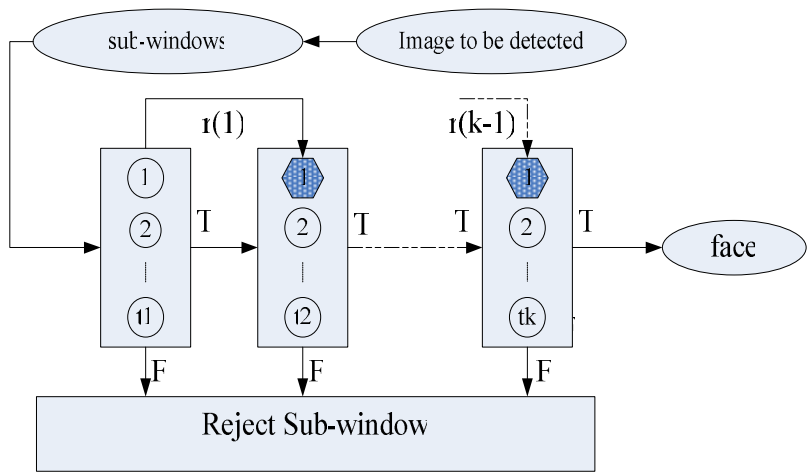

Figure 2. The schematic depiction of the heuristics-structured cascade.

The heuristics parameter $r(i)$ should have the ability to control the proportion of inheritance and independent. Owning to the abandon process of negative samples, the sample structure of one layer is different from each other. The higher the proportion of samples discarded, the larger the independent part and the smaller the inheritance part should be. Since the positive samples keep unchanged and the negative samples change a lot, the heuristic parameter $r_{k-1}$ in new classifier's training is defined as:

$$
r_{k-1}=\left(f_{k-1}+1\right) / 2
$$

Where $f_{k-1}$ is the false positive rate of the $k-1$ th layer. By adding the previous training results into the current classifier, the training process speeds up and the overall number of weak classifiers reduce. In the heuristics-structured cascade framework, the strong classifier is expressed as:

$$
F_{k}(x)=r_{k-1} F_{k-1}(x)+\sum_{t=1}^{T} a_{t}^{(k)} h_{t}^{(k)}(x)
$$

Where, $F_{k-1}(x)$ is the output function of the $k-1$ th layer, $r_{k-1}$ is the heuristic factor of the $k-1$ th layer. $r_{k-1}$ drops as the FPR of the $k-1$ th classifier drops, which means a significant alteration of the samples. On the contrary, $r_{k-1}$ increases. The evaluation of heuristics-structured cascade is as follows:

1) Given an example $x$, assess the heuristics-structured cascade with $k$ layers.

Repeat for $i=1$ to $k$ :

$$
\text { a). } s C=r_{i-1} \times F_{i-1}+F_{i}
$$

b). If $s c<t h r e s h_{i}$, then exit with negative response.

2) Exit with positive response.

Where $F_{i}$ represents the function of the $i$ th layer, and thresh $_{i}$ represents threshold of $F_{i}$.

\section{EXPERIMENTAL RESULTS}

\section{A. Experiment on training set: the MIT-CBCL dataset}

We use the MIT-CBCL dataset as the training set. All experiments are conducted on the simulation software 
MATLAB R2010a (MathsWorks). The MIT-CBCL dataset contains 2429 face samples and 4548 non-face samples with resolution of $19 \times 19$.We set the face detection rate of each layer as 0.99 . The false positive rates of the layer from first to the ninth are set as $0.6,0.5,0.4,0.4,0.4,0.3,0.3,0.3,0.3$, respectively. After obtaining the detector, we evaluate it over all the samples to obtain a ROC curves. Three methods are tested on all of the samples in the MIT-CBCL dataset, they are classical AdaBoost, AD AdaBoost and HAD-AdaBoost. The false mpositive rate (FPR) curves and the FPR under different sum of weak classifiers over the MIT-CBCL dataset are shown in Fig. 3.

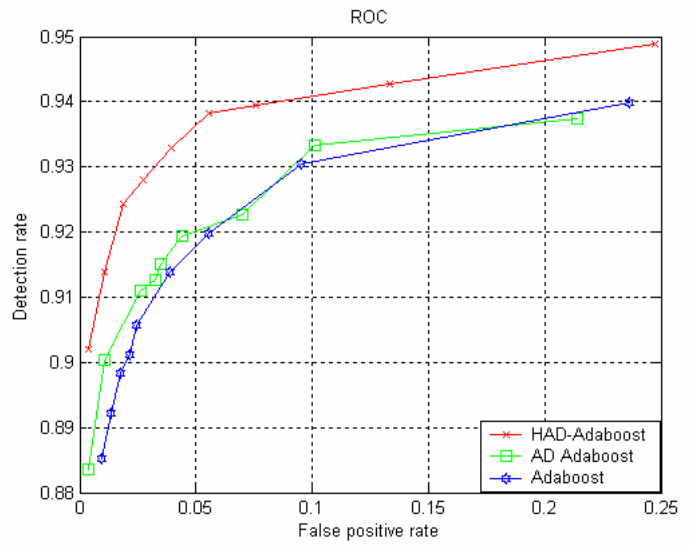

(a)

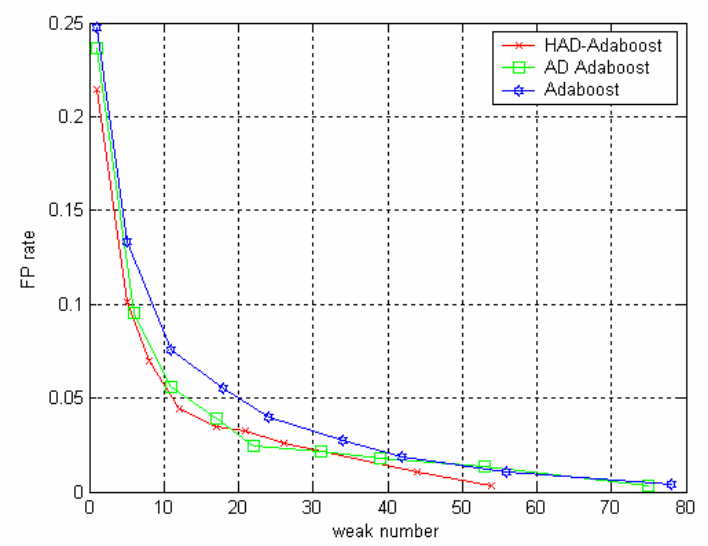

(b)

Figure 3. (a). ROC curve of three algorithms for face detector on the MIT+CBCL dataset. (b). False positive rates for various numbers of weak learners on the MIT+CBCL dataset

In the ROC curve, the detection rate (DR) of HADAdaBoost cascade classifier is 0.9020 and the FPR is 0.0040 while the DR of adaboost is 0.8851 and the FPR is 0.0030 , which means the HAD-AdaBoost algorithm possesses high DR and low FPR. The results clearly demonstrate the effectiveness of HAD-AdaBoost approach. The overall number of weak classifiers and the total training time are shown in Tab. 1:
TABLE I. THE OVERALL NUMBER OF WEAK CLASSIFIERS IN DETECTOR AND TRAINING TIME OF THREE ALGORITHMS.

\begin{tabular}{lll}
\hline \multicolumn{1}{c}{ Algorithm } & \multicolumn{1}{c}{ Weak number } & \multicolumn{1}{c}{ Training time (min) } \\
\hline Classical AdaBoost & 78 & 85.36 \\
Fast AD-AdaBoost & 74 & 67.62 \\
HAD-AdaBoost. & 54 & 53.08 \\
\hline
\end{tabular}

As shown in Tab. 1, the total number of features in HADAdaBoost cascade is 54 while the AdaBoost 78. Therefore, HAD-AdaBoost works with fewer weak classifiers, which greatly cuts down the training time.

\section{B. Experiment on test set: color images set}

In this section, we test the performance of the proposed two-stage face detection method on color image set comprised of digital photos from life and the Internet. There are 250 pictures including 1486 faces in our test set. The performance comparison of the AdaBoost algorithm, HAD-AdaBoost algorithm and the two-stage method on the image set are shown in Tab. 2:

TABLE II. EXPERIMENT ON 250 PICTURES INCLUDING 1486 FACES WITH THREE METHODS.

\begin{tabular}{llll}
\hline \multicolumn{1}{c}{ Method } & \multicolumn{1}{c}{ AdaBoost } & HAD-AdaBoost & The two-stage method \\
\hline Right number & 1289 & 1366 & 1341 \\
Miss number & 185 & 120 & 145 \\
False number & 315 & 174 & 23 \\
DR (\%) & 86.74 & 91.92 & 90.24 \\
FPR (\%) & 19.64 & 11.30 & 1.69 \\
\hline
\end{tabular}

As shown in Tab. 2, the HAD-AdaBoost algorithm is also superior to Adaboost with higher DR and lower FPR. By using the two-stage method, the false detection number of HADAdaBoost reduces obviously due to skin color pretreatment. Two detail examples of the experiments are shown in Fig. 4:

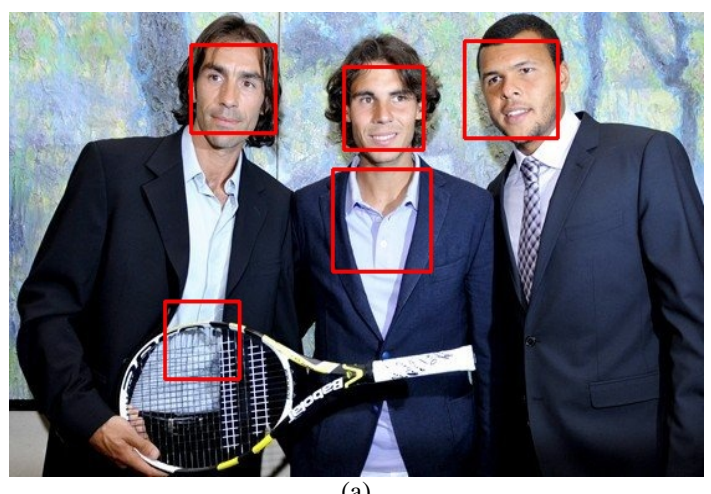

(a) 


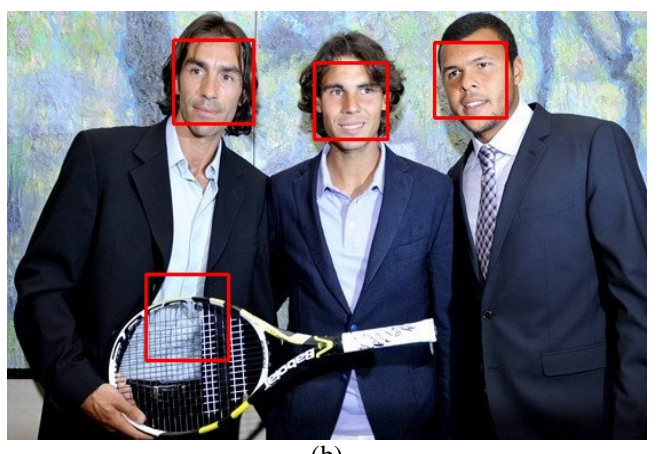

(b)

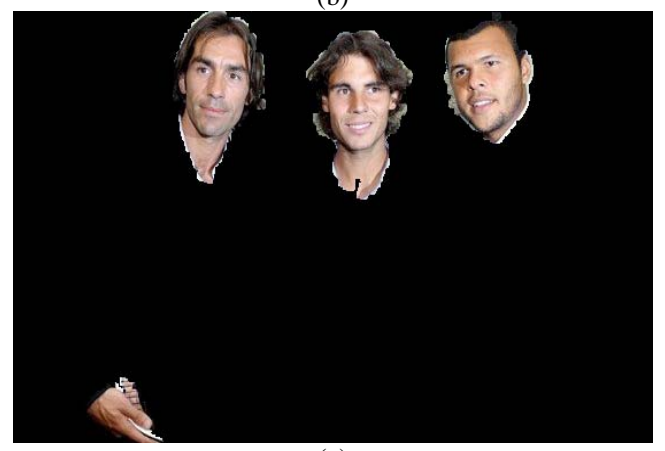

(c)

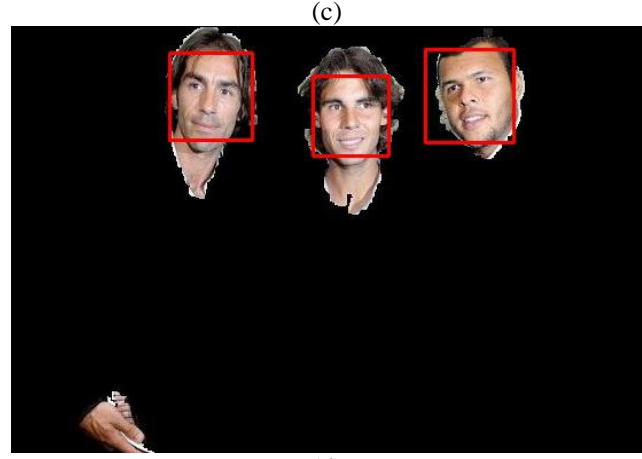

(d)

Figure 4. Comparison of face detection based on three methods-AdaBoost, HAD-AdaBoost and the two-stage method. (a) Detection results by AdaBoost algorithm, (b) Detection results by HAD-AdaBoost algorithm, (c) The candidate skin regions after skin color segmentation, (d) Detection results of the proposed two-stage method.

Both Fig. 4 (a) and Fig. 4 (b) show that some of the backgrounds whose texture similar to face are detected as faces. But in Fig. 4 (b) based on HAD-AdaBoost, the number of fault detected windows is smaller. As shown in Fig. 4 (c), although skin color segmentation can detect all face regions, the result region also contains neck and clothes whose color is similar to skin. In Fig. 4 (d), the proposed method that takes both color and gray features into account leads to a more precise face location.

\section{CONCLUSIONS}

In this paper, a two-stage framework for face detection in color image has been presented. In this framework, skin color segmentation is applied in the first stage to remove most of the background area. An optimized strategy called HAD-AdaBoost to reduce training time and improve classifier performance is applied in the second stage to make a final location. The results on MIT-CBCL dataset and our experimental images demonstrate that the proposed face detection approach enhances the performance of detector for image with complex background.

\section{REFERENCES}

[1] E. Hjelmas, B. K. Low, "Face detection: a survey, Computer Vision and Image Understanding,” vol. 83 (3), pp. 236-274, 2001.

[2] P. Viola, M. Jones, Rapid object detection using a boosted cascade of simple features, in: Proceedings of IEEE International Conference on Computer Vision and Pattern Recognition, pp. 511-518, 2001.

[3] C. Liu, H.Y. Shum, Kullback-Leibler boosting, in: Proceedings of the 2003 IEEE Computer Society Conference on Computer Vision and Pattern Recognition, pp. 587-594, 2003.

[4] S. Z. Li, Z. Q. Zhang, Floatboost learning and statistical face detection, IEEE Transactions on Pattern Analysis and Machine Intelligence, vol. 26 (9), pp. 1112-1123, 2004.

[5] R. Xiao, L. Zhu, H.J. Zhang, Boosting chain learning for object detection, in: Proceedings of the 9th IEEE International Conference on Computer Vision, pp. 709-715, 2003.

[6] Z. B. Guo, Research on the algorithms of fast face detection and feature extraction, Nanjing University of Science and Technology, 2007.

[7] Y. J. Fu, J. W. Li, Rotation invariant multi-View color face detection based on skin color and Adaboost algorithm, in: 2010 International Conference on Biomedical Engineering and Computer Science (ICBECS), pp: 1-5, 2010.

[8] X. S. Gao, X. Z. Zhang and C. Y. Ji, Face detection in color images, Techniques of Automation and Applications, vol. 24 (10), 54-56, 2005.

[9] R. L. Hsu, A. M. Mohamed, A. K. Jain, Face detection in color images, IEEE Transactions on Pattern Analysis and Machine Intelligence, vol. 24 (5), 696-706, 2002.

[10] C. L I, X. Q. Ding, Y. S. Wu, A revised AdaBoost algorithm-AD AdaBoost, Chinese Journal of Computers, vol. 30 (1), 103-109, 2007. 